\title{
On $\mathrm{G}_{0}$ and Cell Cycle Controls
}

\author{
Stephen Cooper
}

Problems and Paradigms is a feature series devoted to discussion of major questions and fundamental issues in biology. In the first article Stephen Cooper discusses one model of cell cycle control and proposes an alternative hypothesis; his treatment is commented upon by Peter Fantes. In the second article, Peter Lachmann describes the basic mechanisms involved in cell injury.

The current model of the animal cell division cycle proposes that the $G_{1}$ phase of the division cycle is the location of most, if not all, of the events regulating the growth and division of animal cells. ${ }^{1}$ Fantes ${ }^{2}$ has reviewed the ideas involved in this current model, and in particular the relationship between the $G_{0}$ phase and the $G_{1}$ period of the division cycle. His point of departure was the work of Zetterberg and Larsson, ${ }^{3}$ who divided the $G_{1}$ period into two parts, a $\mathbf{G}_{1}$ pm (post-mitotic) phase and a $G_{1}$ ps (pre-S) phase. The $G_{1}$ pm period, which in the $3 T 3$ cells studied by Zetterberg and Larsson lasted for 3.5 hours after mitosis, is the only period of the division cycle from which cells can leave the cycle to enter the $G_{0}$ period. As Fantes ${ }^{2}$ points out in his review of this work, the existence of a control point in the $G_{1}$ period fits in with other ideas, such as the unique restriction point of Pardee, which has become 'one of the basic tenets of workers in the field '. ${ }^{2}$ Besides the restriction point, Fantes observes that the idea of a control point in the $G_{1}$ period also receives support from the transition-probability model of Smith and Martin. ${ }^{5}$

I have argued, and will argue here, that there are no $G_{1}$ control points and there is no $G_{1}$-specific regulatory function. In bacteria and animal cells the relevant regulatory events occur continuously during the period between the starts of $S$ phases; there are no $G_{1-}$ specific controls or control points. ${ }^{6-8}$ Here I will apply this alternative view of the division cycle to the results of Zetterberg and Larsson. ${ }^{3}$ I will show not only that their experiments lead to predictions that have not been supported experimentally, but $I$ will also propose a new principle, the Law of Cell Age Order Invariance, against which the model and results of Zetterberg and Larsson can be tested.

What did Zetterberg and Larsson do? The experiment was quite simple. They used a time-lapse video recorder to study cells growing in monolayers. After determining the cycle ages of the growing cells by noting the time since the last mitosis, all of the cells were subjected to varying periods in serumfree medium. The division of the cells was then followed during the treatment and after the serum was replaced. To explain their results with numbers, consider that the interdivision time was $16 \mathrm{~h}$, that the $G_{1}$ period was $7 \mathrm{~h}$, and that the $S, G_{2}$ and $M$ phases accounted for the remaining $9 \mathrm{~h}$. Let us look at the experiment where the cells were starved for $1 \mathrm{~h}$. Zetterberg and Larsson ${ }^{3}$ observed that only cells that were within $3.5 \mathrm{~h}$ of the last mitosis (that is, the youngest cells) were affected in the next division; not only was the division delayed for the time of starvation, but the division was delayed or 'set back' an additional $8 \mathrm{~h}$. Those cells that were older than $3.5 \mathrm{~h}$ and that were now within $12.5 \mathrm{~h}$ of the next mitosis went through the next mitosis on schedule, whether or not the cells were starved for between 1 and $8 \mathrm{~h}$. The cells that were more than $12.5 \mathrm{~h}$ from mitosis (i.e. not older than $3.5 \mathrm{~h}$ ) were 'set back' or delayed in their next scheduled mitosis for at least $8 \mathrm{~h}$.

The model that Zetterberg and Larsson ${ }^{3}$ propose to explain their results is that during the first $3.5 \mathrm{~h}$ the cells are in a $G_{1}$ pm (post-mitotic) phase; when these cells are starved of serum they can enter the $G_{0}$ phase of the cell cycle. Return to the $G_{1}$ period from the $G_{0}$ period takes $8 \mathrm{~h}$ and this accounts for the $8 \mathrm{~h}$ 'setback' in cell division for the younger cells starved for serum. At $3.5 \mathrm{~h}$ after mitosis the cells leave the $G_{1}$ pm phase and enter the $G_{1}$ ps (preS) phase. Cells in this phase go on to divided even if incubated in serum-free medium. Only cells in a particular part of the $G_{1}$ period can enter the $G_{0}$ phase,

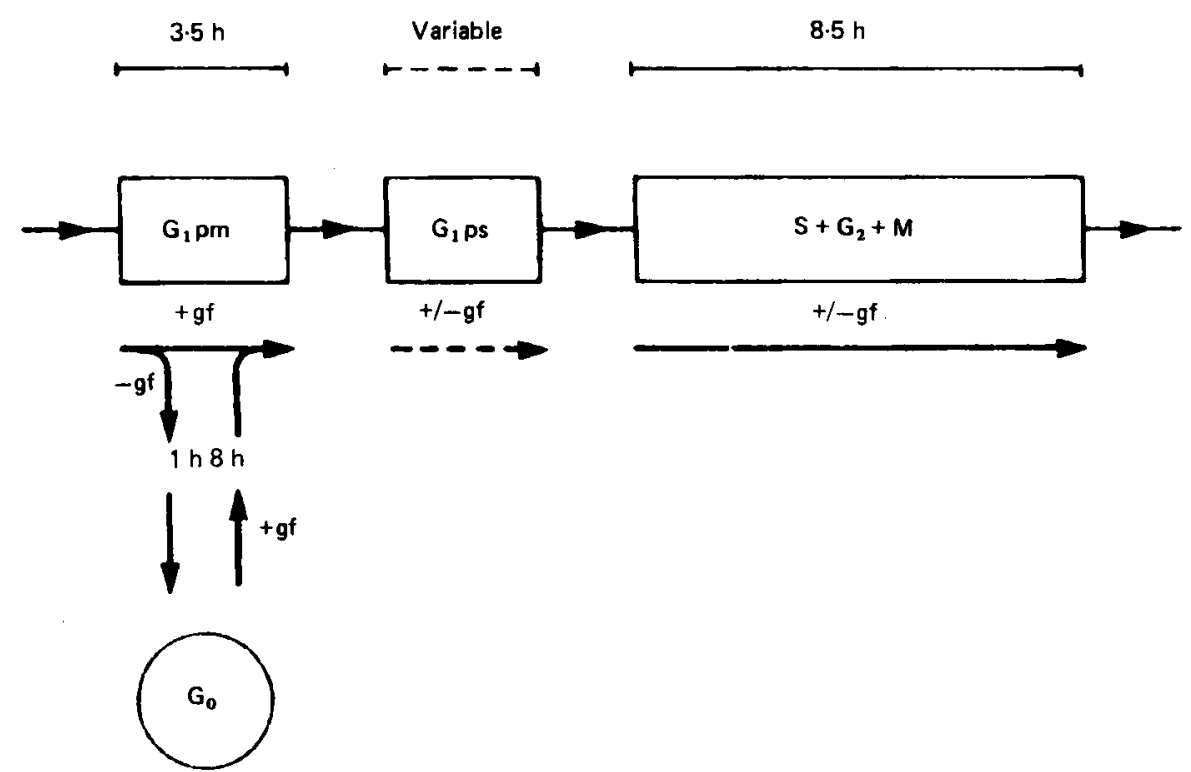

Fig. 1. A schematic description of model of Zetterberg and Larsson (from ref. 3). Their caption to this figure reads: 'During the first 3.5 h after mitosis $\left(G_{1}\right.$ pm) the cell makes the decision whether or not to progress through the cell cycle. This decision depends on the presence of growth factors $(g f)$. If the cell senses a lack of growth factors $(-g f)$ in $G_{1}$ pm it will leave the cell cycle within $15-60$ min and enter a state of quiescence $\left(G_{0}\right)$ from which it takes $8 h$ to re-enter the cycle after the growth factor level in the environment again becomes optimal $(+g f)$ for proliferation. Once the cell has entered $G_{1} p s$ it will eventually initiate DNA synthesis. However, $G_{1}$ ps is highly variable in length and in fact responsible for most of the variability in the duration of the $G_{1}$ and of the whole cell cycle.' 
PROBLEMS AND PARADIGMS

and there is a point in $G_{1}$ that is now defined by this experiment. This control point is a $G_{1}$ regulatory point. The model of Zetterberg and Larsson ${ }^{3}$ is illustrated in Fig. 1.

My analysis is best put in the form of a question: What does the model of Zetterberg and Larsson ${ }^{3}$ imply or predict for subsequent cell cycles? The question is illustrated in the three panels of Fig. 2. In the upper panel, exponential cell growth is illustrated. At time zero there exists, in the exponentially growing culture, cells of different ages. Label these different age groups 1, $2,3,4$, and 5 in order of ascending age. The cells of group 1 are the youngest cells, with ages from 0.0 to 0.2 , the cells of group 2 have ages from 0.2 to 0.4 , and so forth. Although the groups are divided into equal fractions by cell age, there are not equal numbers of cells in each group. There are more cells in the youngest group than in the oldest group because of the exponential age distribution. Because the oldest cells in the culture at time zero will divide to give the cell increment during the first $20 \%$ of the first division cycle, and the youngest cells will divide during the last $20 \%$ of the division cycle, this age distribution allows the exponential increase in cell number.

If we eliminate, for this Gedanken experiment, statistical variability of cell interdivision times, then the predicted order of cell division during unperturbed exponential growth is illustrated in the uppermost panel of Fig. 2. The cell number increases by divisions occurring first in group 5 (the oldest cells at time zero), then groups 4, 3, 2, and 1 ; in the next cycles this order repeats as the daughter and granddaughter cells produced by the first divisions now divide.

What would one expect from the 'setback' model of Zetterberg and Larsson? The predictions of their model, as implied by their results and analysis, are illustrated in the middle panel of Fig. 2. We can approximate the $3.5 \mathrm{~h}$ in the $\mathrm{G}_{1} \mathrm{pm}$ phase as being the first $20 \%$ of the division cycle; that is, the cells in group 1 are in the $G_{1}$ pm phase. Assume that we give a short incubation in serum-free medium so that only cells in group 1 are now 'set back' into $G_{0}$, from which it takes $8 \mathrm{~h}$ to leave. By considering only a short treatment we eliminate problems resulting from cells dividing and newly entering the $G_{1}$ pm state. As the only cells with an affected division pattern are the cells in group 1, groups $5,4,3$, and 2 , comprising $80 \%$ of the division cycle, proceed to divide

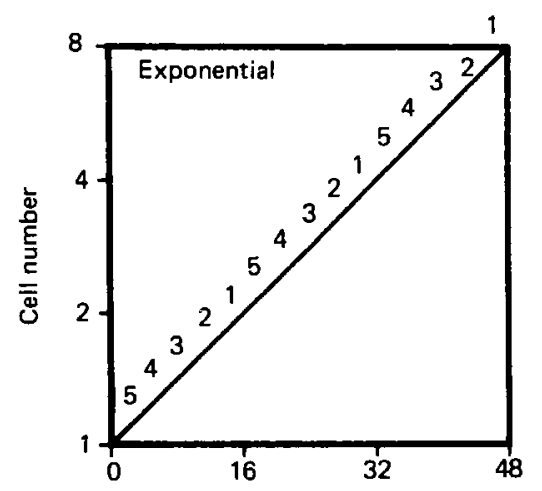

Larsson, the other cells are not able to enter into the $G_{0}$ state and so are unaffected. This is because the other cells are not in the $G_{1}$ pm phase of the division cycle. The daughter cells of groups 5, 4, 3 and 2 continue to divide normally. The expected pattern of cellnumber increase is shown in the middle panel - a cessation of cell division at the end of the first division cycle, and a relative increase in the rate of cell division as the cells of group 1 divide at the same time as the cells of group 3. If the cells in $G_{1}$ pm are truly 'set back', and the other cells are not affected, this is the prediction of the Zetterberg and Larsson model. (N.B. This is not explicitly stated or experimentally studied by Zetterberg and Larsson. Their experiments and model deal only with the first division cycle from the serum starvation. The predictions of their model for the divisions of the second cycle are deduced from their model.) The timelapse video experiments of Zetterberg and Larsson make a prediction of the pattern of cell growth during the subsequent division cycles, and this pattern has never been seen or reported in any of a large amount of work on cells starved in serum. I predict that such a pattern as they would expect will not be observed.

What does the continuum model predict? The continuum model predicts that cells past a point in the division cycle (i.e. the cells in $S$ or $G_{2}$, or $M$ ) are not affected by serum starvation, and will divide on schedule for the remainder of the division cycle. Even with prolonged serum starvation, cells in the $S$, $G_{2}$ and $M$ phases will divide normally to produce cells with a $G_{1}$-DNA content. ${ }^{7}$ (If there is some residual leakage of mass synthesis during serum starvation there would be some 'leakage' of cells in the $G_{1}$ phase into the $S$ phase, and these cells would also subsequently divide; the imperfect inhibition of initiation of $S$ phase could account for the observation of Zetterberg and Larsson that their $G_{1}$ ps phase started somewhat prior to $S$ phase.) But the continuum model predicts that it is impossible to affect the division of the cells in group 1 without also affecting the subsequent (if not the first) divisions of the cells in the other four age groups. The same starvation that caused the delay in cell division of the youngest cells will also cause a delay in the division of the cells in the other groups, but the delay will only be exhibited in the second and succeeding division cycles. Whatever decrease in mass synthesis delayed the division of the youngest cells would also 


\section{PROBLEMS AND PARADIGMS}

affect the other cells because the synthesis of 'initiator' (see ref. 7 for a complete discussion) is occurring at all times during the division cycle, and its synthesis is inhibited in cells of all ages when serum is removed from the growing cells. The continuum model predicts that if there was a setback of the cells of group 1, then there would be setbacks of the other age groups. The pattern of cell increase would be as found in the lower panel of Fig. 2.

There is another way to state this prediction of the continuum model the Law of Cell Age Order Invariance. This law states that it is impossible, by any batch treatment of cells, to alter the order of division of the cells. If a cell A divides before a cell $B$, then the daughters of cell $A$ will divide before the daughters of cell B divide. Statistical variation may lead to changes in age order distribution; cells that have divided just prior to a given cell can have, in any particular instance, their daughter cells divide just after the other daughter cells, but on the average this is not expected. If a cell A divides just prior to another cell $B$, it is possible that the daughters of cell A may divide just after the daughters of cell $B$, but on the average the daughters of cell $A$ will divide before the daughters of cell $B$.

A result not in accord with the Law of Cell Age Order Invariance would be if cells were given a treatment (i.e. a batch treatment of the culture and not any selective treatment of particular cells) that would cause the daughters of cell $\mathrm{A}$ to divide at the same time, or even after, the daughters of cell $B$. If one were to be able to make the two cells divide at the same time, and to now act in the same way with regard to division cycle in subsequent cell cycles, one would be able to synchronize cells by a batch treatment. But the continuum model states that it is impossible to truly synchronize cells in a culture by any batch treatment. ${ }^{7,10}$ It may be difficult to accept such a notion, when there has been so much effort expended on synchronizing cells by various batch treatments - heat shocks, thymidine treatments, starvations, etc.-but in practice all such efforts have not succeeded in producing synchronized cells. (The proof of this Law or principle is beyond the scope of this short article, but it is based on the idea that as there are no cell-cycle-specific regulatory points, there is no treatment of cells of a particular age; all cells are affected equally, and therefore the cell age order is preserved under any batch treatment of cells in culture.)
The Law or Principle of Cell Age Order Invariance is violated by the model of Zetterberg and Larsson, as can be seen by the predictions illustrated in Fig. 2. In the second division cycle the cells of group 1 divide at the same time as the cells of group 3 and after the cells of groups 4 and 5 . Although it is unusual, in biology, to test an experimental result against general principles, this is more common in physics. For example, if someone published a paper on a perpetual-motion machine we would immediately criticize it by citing the Second Law of Thermodynamics. As cell biology matures there will arise various principles that may be used to test experimental results. I suggest that the Law of Cell Age Order Invariance is one of them. The predictions it makes are clear, and if my interpretation of the model of Zetterberg and Larsson ${ }^{3}$ is correct, their model violates this principle.

The $\mathrm{G}_{0}$ concept has been examined before and found wanting. ${ }^{7}$ The fact that it continues to dominate the field of cell-cycle biology is merely testimony to the attractiveness of its siren call, and not any indication that it is true. The alternative idea, the continuum model, which proposes that there are no $G_{1}$ specific regulatory points or events or states, can explain the data better, more clearly, and more simply, than the $G_{0}$ model.

\section{REFERENCES}

1 Baserga, R. (1985). Biology of Cell Reproduction. Harvard University Press, Cambridge, Massachusetts. 2 FANTES, P. (1986). Growth factors, $G_{0}$ and cell cycle controls. Bio Essays 4, 32-33.

3 ZeTterberG, A. \& Larsson, O. (1985). Kinetic analysis of regulatory events in $G_{1}$ leading to proliferation or quiescence of Swiss 3 T3 cells. Proc. Natl. Acad. Sci. USA 82, 5365-5369.

4 PARDEE, A. B. (1974). A restriction point for control of normal animal cell proliferation. Proc. Natl. Acad. Sci. USA 71, 1286-1290.

5 Smith, J. A. \& Martin, L. (1973). Do cells cycle? Proc. Natl. Acad. Sci. USA 70, 1263-1267.

6 CoOPER, S. (1979). A unifying model for the $G_{1}$ period of prokaryotes and eukaryotes. Nature 280, 17-19.

7 COOPER, S. $(1982 a)$. The continuum model: application to $G_{1}-$ arrest and $G_{0}$. In Cell Growth (ed. C. Nicolini), pp. 315-336. Plenum, New York.

8 COOPER, S. (1982). The continuum model: statistical implications. J. Theor. Biol. 94, 783-800.

9 Helmstetter, C. E., CoOper, S., Pierucci, O. \& ReVELAS, E. (1968). Cold Spring Harbor Symp. Quant. Biol. 33, 809-822.

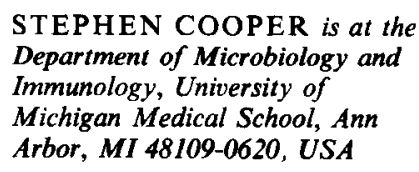

STEPHEN COOPER is at the Department of Microbiology and Immunology, University of Michigan Medical School, Ann Arbor, MI 48109-0620, USA

\section{Peter Fantes comments:}

The view of the cell cycle presented and argued by Cooper ${ }^{1}$ differs in a fundamental way from the conventional view. The commonly accepted view is that the $G_{1}$ period has a definite beginning, and ends at the start of the $S$ phase. The textbook picture is that the events that lead up to $S$ phase are started at the end of mitosis, and are confined to $G_{1}$ (in its strict sense of the interval between mitosis and $S$ phase). Modifications such as in the twotransition probability model ${ }^{2}$ allow for some overlap of cycles so that the train of events leading to the $S$ phase can be started substantially before the previous mitosis, but even here a discrete initiation event (albeit one which is probabilistically controlled) is required.

Cooper's 'Continuum Model'3, on the other hand, states that the $G_{1}$ period has no defined beginning, but rather that processes take place continuously, such as the synthesis of an initiator with special properties, ${ }^{4}$ and culminate in the initiation of the $S$ phase. A consequence of this hypothesis is that any treatment that affects progress through $G_{1}$, or the transition from $G_{1}$ to the $S$ phase, necessarily affects all cells ${ }^{1}$. The effect of the treatment may show up most obviously in a subset of cells: in Zetterberg and Larsson's experiments, ${ }^{5}$ cells in the $G_{1}$ pm interval (using their terminology) are most noticeably affected by pulses of serum starvation. These cells undergo a delay to mitosis that is considerably longer than the duration of the starvation. Cooper $^{1}$ argues that if his model is correct, then cells other than these are also delayed, and that the predicted cell-number rise will be interrupted by an $8 \mathrm{~h}$ period during which no divisions will occur (fig. 2, lower panel, in ref. 1). Some divisions in the population will be 'lost', and the total cell number curve will never again be coincident with that of the unperturbed population, but will be parallel to it. He argues that, in contrast, the Zetterberg and Larsson model predicts, firstly, that the step-like pattern of number increase in the first cycle will be repeated in subsequent generations and, secondly, that the order in which cohorts of perturbed cells divide will be altered in the first and subsequent generations (fig. 2, middle panel, in ref. 1). This predicts that no divisions are 'lost', apart from, perhaps, those of the delayed cohort.

Cooper's first argument ignores the possibility that delayed cells might 'catch up' with the rest of the population in subsequent cycles. Larsson, 
PROBLEMS AND PARADIGMS

Zetterberg, and Engstrom ${ }^{6}$ report in a subsequent paper, not discussed by Cooper, ${ }^{1}$ that cells delayed for one mitosis by starvation undergo a shortened second cycle. In other words, there is evidence for cycle time homeostasis. The extent of shortening is less than the original delay, as seen in other recovering systems, but it is possible that these cells will catch up over several cycles with untreated control cells. In this case the cell-number curve for starved cells may eventually coincide with the control curve, and not resemble that predicted by Cooper.

However, Larsson et al. ${ }^{6}$ also show that cells too late in the starvation cycle to be mitotically delayed in the first cycle have an extended second cycle. This is, paradoxically, in agreement with Cooper's contention that cells other than the delayed $G_{1}$ pm cells are affected by serum starvation. The kinetics show that cells later in the first cycle (and therefore nearer to the next $\mathrm{S}$ phase) at the time of starvation are more delayed in the second cycle.

It is therefore not clear whether Cooper's proposal ${ }^{1}$ is consistent with the combined data of Larsson et al. ${ }^{6}$ The most extreme expression of Cooper's ideas, the 'Law of Cell Age Invariance', which states that no batch treatment of cells can reverse their order of division, is attractive as a general rule, but remains to be critically tested by experiment. A reanalysis of Larsson's observations and their presentation in a way suitable for Cooper's analysis might be highly informative.

\section{REFERENCES}

1 COOPER, S. (1987). (Accompanying article) BioEssays 7, 220-222.

2 BROOKs, R. F., BenNetT, D. C. \& SMith, J. A. (1980). Mammalian cell cycles need two random transitions. Cell 19, 493-504.

3 COOPER, S. (1979). A unifying model for the $G_{1}$ period of prokaryotes and eukaryotes. Nature 280, 17-19. 4 COOPER, S. (1982). The continuum model: application to $G_{1}$ - arrest and $G_{0}$. In Cell Growth (ed. C. Nicolini), pp. 315-336. Plenum, New York.

5 ZetTer berG, A. \& LARSSON, O. (1985). Kinetic analysis of regulatory events in $G_{1}$ leading to proliferation or quiescence of Swiss 3T3 cells. Proc. Natl. Acad. Sci. USA 82, 5365-5369.

6 Larsson, O., Zetterberg, A. \& Engstrom, W. (1985). Consequences of parental exposure to serum-free medium for progeny cell division. J. Cell Sci. 75, 259-268.

PETER FANTES is at the Department of Zoology, University of Edinburgh, West Mains Rd., Edinburgh EH9 $3 J T$, UK

\title{
Plugs, Burns and Poisons
}

\author{
Peter J. Lachmann
}

This paper is based on the first $R$. $R$. Porter Memorial Lecture given at St George's Hospital Medical School in March 1986.

The title of this paper is meant to encapsulate the three major mechanisms by which eukaryotic cells can be killed by other eukaryotic cells and by that unique plasma cytolytic system, the membrane attack complex of complement.

The term 'plug' is used to describe cytolytic mechanisms associated with the insertion into the target cell membrane of extrinsic material derived either from the killer cell or from the complement system. I have preferred the somewhat non-specific and possibly imprecise term, 'plug' to such more precise terms as pore-forming agent or channel-former not only because it is shorter but because these latter names generally denote a channel or pore formed through the inserted protein; i.e. the insert is hollow and allows leakage through its centre. The term plug, however, can equally denote what has been more correctly described as a 'leaky patch', where the insert is solid and causes leakage not through its centre but around its periphery where there is distortion of the lipid bilayer.
The question whether the much studied complement lytic lesion is a solid plug (leaky patch) or a hollow plug (poreformer) has caused a long-standing and still incompletely resolved controversy.

The term 'burn' is used to denote the damage to cells produced by oxygen metabolites. The generation of active metabolites of oxygen by the superoxide burst is implicated in a variety of pathological mechanisms and undoubtedly can cause cell death. Here again however, the precise biochemistry, the nature of the toxic end-product and the target of the essential lytic event are still subjects of some dispute. The term 'burn' was suggested by the idea that the final lesion was the peroxidation of cell membrane lipids, but the term is to be understood as encompassing all sorts of damage produced by a variety of toxic oxygen metabolites.

The term 'poison' is even more general and embraces all or any product produced by the killer cell that is capable of killing cells by mechanisms other than those given in the first two mechanisms. However, there are now some well characterized toxic molecules that are used in cytotoxic mechanisms. The ones which are most clearly described are tumour necrosis factor and lymphotoxin (TNF- $\alpha$ and TNF- $\beta$ ); cytokines whose role in, for example, ADCC by monocytes is well established and whose structure is now fully known but whose exact mechanism in cytotoxicity is still under investigation. Other toxic factors have been described in some profusion. Some such as arginase starve the target cell to death by depriving it of a specific essential nutrient. Others such as the proteases described in cytotoxic $T$ cells have mechanisms of actions which are not clear.

In this paper I propose to review - in a somewhat slanted way - the current state of knowledge of these cytotoxic mechanisms and to make the particular point that there is growing evidence that these mechanisms are used in association with one another and that it may well turn out to be the case that most cytotoxic cells can employ more than one cytotoxic mechanism. Thus, lymphocytes can both form plugs and produce poisons. Monocytes similarly, can produce both poisons and burns, and the eosinophil is known to use plugs and burns.

The best studied cytotoxic mechanism is that of the complement system which may be unusual in that it relies wholly on the 'plug'; this may have mislead some research workers into taking unnecessarily polarized attitudes 